\title{
Streamlined percutaneous atrial septal defect closure in adults
}

\author{
Selma Arı®, Hasan Arı®, Veysi Can®, Sencer Çamcı®, Mehmet Melek® \\ Department of Cardiology, Bursa Yüksek İhtisas Training and Research Hospital, Bursa, Turkey
}

\begin{abstract}
Objectives: The aim of the study to evaluate the safety and efficacy of the transthoracic echocardiography (TTE) guided secundum atrial septal defect (ASD) closure without balloon sizing, sedation or general anesthesia in adults.

Methods: We retrospectively evaluated 200 secundum ASD closure patients in the tertiary cardiology center. Transesophageal echocardiography (TEE) was performed to all the patients at least one day before the intervention by the procedure operators. The patients who were closed with a cribriform device, using more than one device, with insufficient rim $(<5 \mathrm{~mm})$ (other than the anterior superior rim (aortic rim)), totally flail, and complex interatrial septum anatomy were excluded from the analysis. The size of the ASD closure device was chosen according to the largest diameter measured by TEE. ASD device was selected as $4 \mathrm{~mm}$ larger in patients without anterior superior rim and $2 \mathrm{~mm}$ larger in other patients than the largest diameter measured in 2D-TEE.
\end{abstract}

Results: In the remaining 166 patients, the procedure was performed with TTE and fluoroscopy guidance without balloon sizing, sedation or general anesthesia. The procedure was performed through right femoral vein. The patients age: $38.56 \pm 14.72$, gender: 57 male, 109 female, ASD size: $18.88 \pm 5.99 \mathrm{~mm}$, anterior superior rim: $5.30 \pm 4.04 \mathrm{~mm}$, anterior inferior rim: $14.22 \pm 6.46 \mathrm{~mm}$, posterior superior rim: $17.16 \pm 4.96$ $\mathrm{mm}$, posterior inferior rim: $16.67 \pm 7.48 \mathrm{~mm}$. ASD device size: $23.74 \pm 6.59 \mathrm{~mm}$. The procedure success rate was $98,1 \%$ (163 patients). The complications; 1 patient device embolised, 2 patients device was not placed in the correct position by TTE.

Conclusions: TTE and fluoroscopy-guided secundum ASD closure without balloon sizing, sedation or general anesthesia by experienced operators is a safe and effective procedure.

Keywords: Atrial septal defect, transthoracic echocardiography, transesophageal echocardiography, closure, sedation

Tn adults, the atrial septal defect (ASD) is the most seen congenital heart disease after bicuspid aortic valve. Four different types of ASDs have been described. Secundum ASD is the most common type and is seen in $80 \%$. Percutaneous ASD closure, which was first performed in 1974, has become the standard treatment in appropriate cases today [1]. At the pres- ent, surgical treatment of secundum ASD patients is performed only in cases not suitable for percutaneous treatment [2]. The procedure of percutaneous ASD closure is generally performed with general anesthesia and TEE or ICE guidance. Selection of the appropriate device size for ASD closure is done by the balloon sizing method. But, in recent years, percutaneous ASD 
closure is performed with TTE and sedation in suitable cases [3].

In this study, we aimed to evaluate the safety and effectiveness of percutaneous ASD closure under local anesthesia, concomitant TTE and without balloon sizing.

\section{METHODS}

We retrospectively evaluated 200 patients who were performed percutaneous closure procedure in our clinic and we included 166 patients who underwent the percutaneous closure procedure with TTE. Patients with complex Secundum ASD, need general anesthesia, need sedation and TEE were not included our study group.

Secundum ASD which closed with TTE and patients with adequate rims except the aortic rim are inclusion criteria for our study. Using more than one device, cribriform ASD, inadequate rims except aortic rim, secundum ASD which closed with TEE, the procedure performed with balloon sizing or under general anesthesia and complex atrial septum are the exclusion criteria for our study. General anesthesia was performed in patients with complex ASD closure procedure (using more than one device, insufficient rims with large ASD, etc.). If the patients need TEE guidance for ASD closure we used sedation. We performed the procedure with local anesthesia for all other patients.

\section{Echocardiography procedure}

TTE was performed to all ASD patients before the procedure. Echocardiographic measurements of the patients were done with X5-1 probe (EPİQ 7, Philips,

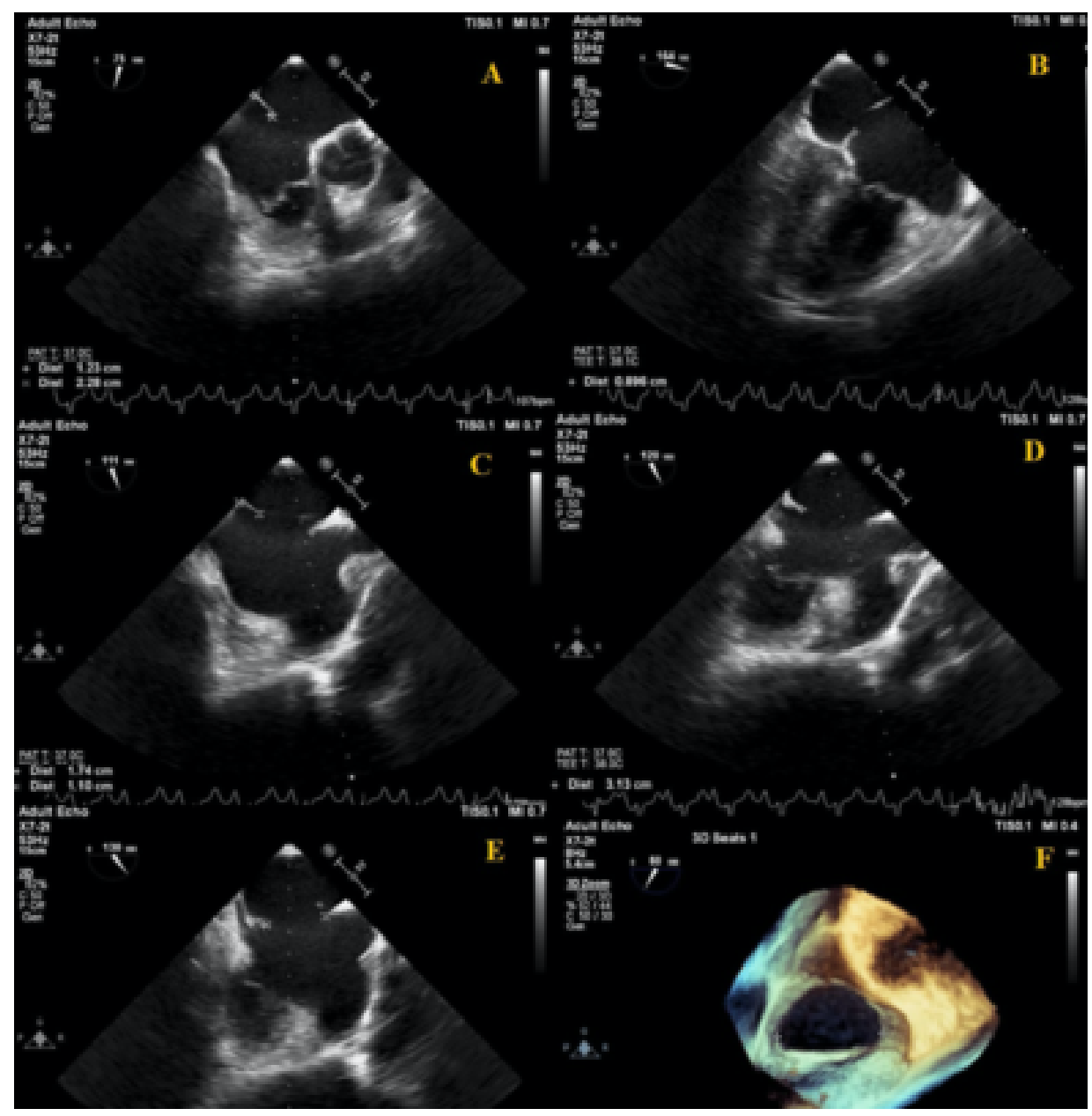

Fig. 1. (A) Short axis image of ASD in TEE, anterior superior rim (aortic rim); (B) Four chamber image of ASD in TEE, anterior inferior rim; (C) Bi-caval image of ASD in TEE, posterior süperior and posterior inferior rim; (D) Maximal ASD diameter in TEE; (E) ASD diameter and coronary sinus rim; (F) 3D image of ASD in TEE. 
Nederland). Heart diameters and functions were measured. Qp/Qs value calculated. One day before the procedure, TEE was performed to all the patients with X7-2t probe (EPIQQ 7, Philips, Nederland). In this process, ASD diameter and rims were measured, and their suitability for closure was evaluated. In the short axis image ASD diameter, anterior superior rim, posterior rim and narrowest atrial septum diameter, in the bicaval image ASD diameter, posterior superior rim and inferior rim, in four chamber image ASD diameter, anterior inferior rim were measured (Fig. 1). The widest diameter of ASD was measured at atrial enddiastole.

\section{Closing procedure and device selection}

The patients were taken to the hemodynami laboratory. Sheat was placed in the right femoral vein after local anesthesia. Fluoroscopy and TTE-guided, MP catheter was passed from ASD to the left atrium and the catheter was advanced to the pulmonary vein. 0,035-inch guidewire was placed into the pulmonary vein through the catheter. The delivery catheter, suitable for the ASD device was advanced to the left atrium over this guidewire. After that, ASD device which determined according to TEE measurements performed one day before the procedure was placed in the appropriate position under the guidance of TTE and fluoroscopy and the device was released. ASD device size was determined according to the largest ASD diameter which measured by 2D TEE. If the aortic rim was adequated together with other rims of the patient and septum was not flail, $2 \mathrm{~mm}$ wider device than the maximal ASD diameter which measured by TEE was selected, whereas $4 \mathrm{~mm}$ wider device was selected in patient with the insufficient aortic rim. If the atrial septum is flail and aortic rim is sufficient, we chosed $4 \mathrm{~mm}$ wider device than the measured defect diameter. We chosed 6-8 mm wider device, in patients with flail atrial septum and insufficient aortic rim. We considered the narrowest diameter of the septum while choosing the device size.

One day before the procedure, aspirin $100 \mathrm{mg} 1 \times 1$ and clopidogrel $75 \mathrm{mg} 1 \times 1$ treatment were started and this treatment was used for six months. Before the procedure, 2 grams of ampicillin were given to the patients. Endocarditis prophylaxis was recommended for patients who underwent an interventional procedure for 6 months after ASD closure.

\section{Statistical Analysis}

Statistical analysis was performed with SPSS (Statistical Package for the Social Sciences ver. 23, SPSS Inc, Chicago, Illinois, USA) computer program. Descriptive analysis was used to calculating to mean value of the continuous variables. Frequency analysis was used for calculating the categorical variables. Numerical variables were expressed as mean \pm standard deviation and categorical variables were expressed as mean percentages.

\section{RESULTS}

Two hundred patients were evaluated for this retrospective study and 34 of them were excluded. Cribriform ASD device was used in 2 of these 34 patients. Two devices were used in 2 patients due to two ASD defects. 30 patients were excluded from the

\section{Table 1. Demographic and biochemical} characteristics of the patients

\begin{tabular}{lc}
\hline Variable & Data \\
\hline Age (year) & $38.56 \pm 14.72$ \\
Gender, $\mathrm{n}(\%)$ & $109(65.6)$ \\
Female & $57(34.4)$ \\
Male & $24.82 \pm 4.87$ \\
BMI $(\mathrm{kg} / \mathrm{m} 2)$ & $73.50 \pm 9.80$ \\
Heart rate $(\mathrm{ppm})$ & $112.93 \pm 20.31$ \\
SBP $(\mathrm{mmHg})$ & $69.27 \pm 11.56$ \\
DBP $(\mathrm{mmHg})$ & \\
Rhythm, n (\%) & $157(94.6)$ \\
SR & $9(5.4)$ \\
AF & \\
Laboratory parameters & $7.18 \pm 2.31$ \\
WBC (103/dL) & $13.02 \pm 1.71$ \\
Hemoglobin (gr/dL) & $224.07 \pm 42.62$ \\
Platelet (103/dL) & $96.20 \pm 30.43$ \\
Glukoz (mg/dL) & $25.57 \pm 9.81$ \\
\hline Urea (mg/dL) & $0.76 \pm 0.17$ \\
\hline Creatinine (mg/dL) & \\
\hline D & \\
\hline
\end{tabular}

Data are shown as mean \pm standard deviation or $\mathrm{n}(\%) . \mathrm{AF}=$ Atrial fibrillation, $\mathrm{BMI}=$ Body mass index, $\mathrm{DBP}=$ Diastolic blood pressure, $\mathrm{SBP}=$ Systolic blood pressure, $\mathrm{SR}=$ Sinus rhythm, WBC = White blood cell. 
study because of their rims were insufficient. The reason for the exclusion of these complicated ASD patients was that the percutaneous ASD closure performed with TEE guidance.

Remaining 166 patients were evaluated. Percutaneous closure was performed successfully in 163 of 166 patients $(98.19 \%)$ by TTE.

TEE had to be performed in 2 patients, because correct position could not be provided or could not be sure that it was in the correct position with TTE. ASD device was successfully placed in both patients with TEE. ASD device was embolized to right ventricle in one patient due to flail rim.
Our patients were between 14-72 years old and ASD device diameters were between 12-36 $\mathrm{mm}$. Demographic and laboratory features of our patients are shown in Table 1. TTE, TEE data and ASD closing device features of our patients are shown in Table 2 .

\section{DISCUSSION}

We found that in adult population TTE and fluoroscopy-guided percutaneous secundum ASD closure without balloon sizing, sedation or general anesthesia at experienced operators is a safe and effective proce-

Table 2. Echocardiographic and device parameters of the patients

\begin{tabular}{lc}
\hline Variable & Data \\
\hline TTE parameters & \\
EF $(\%)$ & $62.68 \pm 5.61$ \\
\hline LVEDD (mm) & $42.94 \pm 4.48$ \\
\hline LVESD (mm) & $25.43 \pm 3.60$ \\
\hline RV end-diastolic diameter, mm & $40.89 \pm 4.27$ \\
Left atrium (mm) & $36.36 \pm 6.23$ \\
\hline Right atrial mediolateral diameter (mm) & $38.49 \pm 7.57$ \\
sPAP (mmHg) & $38.65 \pm 9.90$ \\
Qp/Qs & $2.2 \pm 0.6$ \\
\hline TEE parameters & \\
\hline Maxilmal ASD diameter (mm) & $18.88 \pm 5.99$ \\
Narrowest septum diameter (mm) & $39.83 \pm 7.40$ \\
\hline Anterior superior rim (mm) & $5.30 \pm 4.04$ \\
\hline Anterior inferior rim (mm) & $14.22 \pm 6.46$ \\
\hline Posterior uperior rim (mm) & $17.16 \pm 4.96$ \\
\hline Posterior inferior rim (mm) & $16.67 \pm 7.48$ \\
\hline Device parameters & \\
\hline Type, $n(\%)$ & \\
\hline MemoPart & $65(39.15)$ \\
\hline Amplatzer & $47(28.31)$ \\
\hline Lifetech Cera & $37(22.28)$ \\
\hline OtherS & $17(10.24)$ \\
\hline Device size (mm) & $23.74 \pm 6.59$ \\
\hline Device size / septum size ratio & $0.58 \pm 0.15$ \\
\hline
\end{tabular}

Data are shown as mean \pm standard deviation or $\mathrm{n}(\%) . \mathrm{ASD}=$ Atrial septal defect, $\mathrm{EF}=$ Ejection fraction, LVEDD = Left ventricular end-diastolic dimension, LVESD = Left ventricular end-systholic dimension, sPAP $=$ Systholic pulmonary artery pressure, TEE $=$ Transesophageal echocardiography, $\mathrm{TTE}=$ Transthoracic echocardiography 
dure.

Previously, especially in pediatric patients, percutaneous ASD closure was performed with TTE [4]. There are some study showing that ASD closure was performed with TTE in adults [3]. Today, success in ASD closure is over 98\% [5]. Success in this case has been achieved concomitant with TEE and balloon sizing. In our cases, this success was achieved with TTE.

Standard recommendation in ASD closing processes in $<11 \mathrm{~mm}$ defects, it is the closure of the defect with a 2-4 mm wider device than the defect diameter measured by TTE, without balloon sizing. These types defects are generally seen in pediatric patients and TTE imaging is usually enough. For 11$24 \mathrm{~mm}$ sized defects, the defect can be closed with a device at the measured maximum defect diameter by balloon sizing method (fluoroscopy; LAO at $15^{\circ}$ and TEE). For 24-32 mm defects, the defect can be closed with $2 \mathrm{~mm}$ wider device than stretched defect diameter by measurement balloon sizing method (fluoroscopy; LAO at $15^{\circ}$ and TEE). Defects with inadequate aortic rim can be closed with $4 \mathrm{~mm}$ wider device than the stretched defect diameter by measurement balloon sizing method and in these defects, atrium roof rim and posterior rim should be sufficient.

The ratio of device size and septum size is important for ASD closure [6]. If the ratio of device size to the septum size is $<0.58$, complications that may occur after the procedure will be less common [6]. If this rate increases, the risk of developing arrhythmia increases after the procedure (such as AF, SVT, AV block). In our study, the ratio device to defect diameter is 0.58 . Choosing a large device also creates a large device area in the heart by making a mushroom effect and that prolongs the epithelialization time of the device. These problems occur in devices with two standard discs and one waist part. Today, different shaped and more elastic devices are available. With these devices, possible complications are tried to be minimized $[2,7]$.

Our patients consisted of the adult population and there was no ASD smaller than $10 \mathrm{~mm}$. In our patients, we performed TEE the day before the procedure and we selected the device according to this TEE measurement. TEE imaging performed by operator who had done ASD closure procedure. ASD diameters change in the systolic and diastolic phase, for this the ASD diameter should be measured at the atrial end- diastole and at the widest defect size [8]. We determined the device size according to the widest ASD diameter which measured atrial end-diastole with TEE. One of the factors in the choice of device size is the narrowest diameter of the atrial septum in the enddiastole. The diameter in the short axis imaging is the narrowest diameter. The rims of ASD closure devices are 7-8 $\mathrm{mm}$ and the device size is $14-16 \mathrm{~mm}$ wider than the waist of the device in total. That is, it should be evaluated whether the ASD devices to be placed will fit into the narrowest atrial septum, taking in to account the wing lengths.

As seen from our successful procedure results, it is seen that our device selection is correct. The important thing is that operator should be experienced in TEE imaging, and the pre-procedure imaging should be performed by the operator. We must say that 2D images provide more accurate information when choosing the device size. Because 3D images are reconstructed images and defect boundaries may not be clearly visible especially in flail and thin septum, they should not be used in device size selection. However, 3D images provide very clear information for understanding the shape of the ASD and anatomy of the septum. This imaging modality will be very helpful in percutaneous ASD closure procedures, especially in ASD patients with complex anatomy. Two-plane modality in 3D probes also facilities the evaluation of ASD.

Operator experience is important for the correct device and diameter selection in ASD closure. Positioning the device parallel to the septum during the closing procedure will provide to settle the device correctly. Some devices and delivery systems have made changes in the delivery system, like moving head for this purpose [7]. In standard devices, the operator tries to provide a parallel position to the septum by changing the delivery angles. Various maneuvers, techniques, and assistive devices can be used to position the device in the correct position. These maneuvers, techniques and assistive device are used especially in large and complex ASD.

Maneuvers and techniques are clockwise rotation maneuvers, tulip bud technique, Greek maneuver, right and left upper pulmonary vein technique, left atrium roof technique. Assistive device techniques are Hausdorf sheath technique, SSH technique or Kutty's method, Boosfeld-Spies technique, wire assisted tech- 
nique, dilatator or catheter asisted technique, balon asisted technique and Nounou technique.

Clockwise rotation maneuvers; the device, which is advanced to the left atrium, is directed to the right upper pulmonary vein with clockwise rotation, after that opening the left disk of ASD device in the left atrium and then pulling the delivery system to open the right disk of ASD device in right atrium. It is the first maneuver to make the ASD device parallel to the septum in which patients without aortic rim [9].

Tulip bud technique; the device is protruded to form a tulip bud in the left atrium. It aligns adjacent to and inplane of ASD. The delivery system is withdrawn in quick succession [9].

Greek maneuvers; the left disc and 2/3 of the right disc of the device are opened in the left atrium, the delivery system and ASD device are positioned by pulling back to the septum and the right disc is opened. With this maneuver, the left disc protrusion is prevented from into the right atrium [10].

Right and left upper pulmonary vein technique; this technique is applied by opening the left atrium disc in the upper left or upper right pulmonary vein then extending the device, after that opening the right atrium disc in the right atrium, the device is pulled back to bring the left atrium disc to the correct position. If the left disc which opened in the pulmonary vein can not be opened by pulling, the left disc can be released by pushing forward with the right atrial disc delivery cable (contrarian technique) [9]. The left upper pulmonary vein technique is applied in patient with large ASD who without aortic rim and other rims are flail. The right upper pulmonary vein technique is applied in patients with inadequate aortic and posterior rim, with aneurysmatic inter-atrial septum, with flail rims, and patients with a not too big ASD diameter [9].

Left atrium roof technique; the left disc is opened between the interatrial septum and the upper left pulmonary vein in the left atrium roof, the device is extended and the right atrial disc opens in the right atrium then the device is pulled and placed at the interatrial septum from the left atrium roof. It is used in patients with small left atrium and with both aortic and posterior rim are inadequate [9].

Hausdorf sheath technique; it is the use of this delivery system (Hausdorf sheath), the end of which is shaped. This technique uses for large ASD with deficiency of anterior and/or aortic rim [11].

Straight Side-Hole sheath technique or Kutty's method; Mullin sheath end of side is cut to be atraumatic and angled (Uses Mullins Transeptal sheath, 1 to $2 \mathrm{~F}$ sizes larger than the minimum recommended sheath for ASD device). This method is used in inadequate inferior or anterosuperior (aortic) rims [11, 12]. Boosfeld-Spies technique; in this technique, 12F Cook Mullins-type sheath (Cook Medical) end-side of it is cut to be atraumatic and angled. This sheath is used as a delivery system. This technique is used in large and inadequate rim ASD [13].

Wire assisted technique; a wire is used to prevent left atrial disc to protrude into the right atrium. The wire can be inserted from the same delivery sheath or another sheath. It can be performed from the same sheath using thinner wire like 0,018 -inch. This wire must be pulled back before the device is released [14].

Dilatator or catheter assisted technique; a multipurpose/JR/dilator catheter is placed in the left atrium across the ASD to prevents prolapse left atrial disc to the right atrium. This technique is used in large ASD and inadequate anterior, anterior süperior (aortic), and/or posterior rims [15].

Ballon assisted technique; this method needs two entries; first entry is for the delivery system and the second entry is needed to send balloon over the guidewire in which located in the right or left upper pulmonary vein. Firstly, the left disk of the ASD device is opened in the left atrium then the balloon is inflated, and after the left disk remains in the proper position with the balloon, the delivery system is pulled back and the right disk is opened. Balloon prevents the prolapse of the left atrial disc into the right atrium. After the device is positioned, the balloon is deflated and pulled. This technique is used for large ASD with flail and inadequate rims [16].

Nounou technique; the device is positioned using Agilis catheter. $20 \mathrm{~mm}$ and smaller ASD devices can be used with this technique. The reason is that catheter diameter is not suitable for larger devices [17].

\section{Limitations}

The limitations of this study are that the study is done retrospectively and not included a group to compare. However, this study contains a detailed examination and has been done with sufficient number of patients, for this, it is capable of guiding operators 
who will perform a closure procedure.

\section{CONCLUSION}

In suitable cases of adult secundum ASD, percutaneous ASD closure can be performed with TTE and fluoroscopy guidance without balloon sizing and general anesthesia, with expert operators.

\section{Conflict of interest}

The author disclosed no conflict of interest during the preparation or publication of this manuscript.

\section{Financing}

The authors disclosed that they did not receive any grant during conduction or writing of this study.

\section{REFERENCES}

1. King TD, Milk NL. Nonoperative closure of atrial septal defects. Surgery 1974;75:383-8.

2. Abaci A, Unlu S, Alsancak Y, Kaya U, Sezenoz B. Short and long term complications of device closure of atrial septal defect and patent foramen ovale: meta-analysis of 28,142 patients from 203 studies. Catheter Cardiovasc Interv 2013;82:1123-38.

3. Ding C, Chang JK, Lin CC, Wu YJ, Hsieh KS. Efficacy and safety of transthoracic echocardiography alone in transcatheter closure of secundum-type atrial septal defects in adults. Echocardiography 2016;33:579-85.

4. Zaqout M, Suys B, De Wilde H, De Wolf D. Transthoracic echocardiography guidance of transcatheter atrial septal defect closure in children. Pediatr Cardiol 2009;30:992-4.

5. Yang M-C, Wu J-R. Recent review of transcatheter closure of atrial septal defect. Kaohsiung J Med Sci 2018;34:363-9.

6. Jin M, Ding W-H, Wang X-F, Guo B-J, Liang Y-M, Xiao Y-Y, et al. Value of the ratio of occluder versus atrial septal length for predicting arrhythmia occurrence after transcatheter closure in children with ostium secundum atrial septal defect. Chin Med J 2015;128:1574-8.

7. Bissessor N. Current perspectives in percutaneous atrial septal defect closure devices. Med Devices (Auckl) 2015;8:297-303. 8. Silvestry FE, Cohen MS, Armsby LB, Burkule NJ, Fleishman $\mathrm{CE}$, Hijazi ZM, et al. Guidelines for the echocardiographic assessment of atrial septal defect and patent foramen ovale: from the American Society of Echocardiography and Society for Cardiac Angiography and Interventions. J Am Soc Echocardiogr 2015;28:910-58.

9. Dalvi B, Jain S. Atrial septal defect: step-by-step catheter closure. J Struct Heart Dis 2016;2:15-32.

10. Thanopoulos BD, Dardas P, Ninios V, Eleftherakis N, Karanasios E. Transcatheter closure of large atrial septal defects with deficient aortic or posterior rims using the "Greek maneuver". A multicenter study. Int J Cardiol 2013;168:3643-6.

11. Hijazi ZM, Feldman T, Sievert H, Al-Qbandi MHA. Transcatheter closure of ASDs and PFOs: a comprehensive assessment: Cardiotext Publishing, 2010.

12. Kutty S, Asnes JD, Srinath G, Preminger TJ, Prieto LR, Latson LA. Use of a straight, side-hole delivery sheath for improved delivery of Amplatzer ASD occluder. Catheter Cardiovasc Interv 2007;69:15-20.

13. Spies C, Boosfeld C, Schräder R. A modified cook sheath for closure of a large secundum atrial septal defect. Catheter Cardiovasc Interv 2007;70:286-9.

14. Chiam PT, Cohen HA, Ruiz CE. The parallel wire technique for septal defect closure. Catheter Cardiovasc Interv 2008;71:564-7.

15. Wahab HA, Bairam AR, Cao QL, Hijazi ZM. Novel technique to prevent prolapse of the Amplatzer septal occluder through large atrial septal defect. Catheter Cardiovasc Interv 2003;60:543-5.

16. Dalvi BV, Pinto RJ, Gupta A. New technique for device closure of large atrial septal defects. Catheter Cardiovasc Interv 2005;64:102-7.

17. Nounou M, Harrison A, Kern M. A novel technique using a steerable guide catheter to successfully deliver an Amplatzer septal occluder to close an atrial septal defect. Catheter Cardiovasc Interv 2008;72:994-7. 